\title{
Learning Methods of Business Intelligence and Group Related Diagnostics on Patient Management by Using Artificial Dynamic System
}

\author{
Abdulsattar Abdullah Hamad, ${ }^{1}$ Mustafa Mahdi Abdulridha, ${ }^{2}$ Noor Mohammed Kadhim, ${ }^{3}$ \\ S. Pushparaj, ${ }^{4}$ R. Meenakshi, ${ }^{3}$ and Abdelrahman Mohamed Ibrahim ${ }^{5}$ \\ ${ }^{1}$ Department of Mathematics, College of Sciences Tikrit University, Iraq \\ ${ }^{2}$ School of Economics, Madurai Kamaraj University, India \\ ${ }^{3}$ Department of Education, Madurai Kamaraj University, Madurai, 625021 Tamil Nadu, India \\ ${ }^{4}$ School of Economics Madurai Kamaraj University, Madurai, 625021 Tamil Nadu, India \\ ${ }^{5}$ Accounting and Financial Management, School of Management Studies, University of Khartoum, Sudan
}

Correspondence should be addressed to Abdelrahman Mohamed Ibrahim; amibrahim@uofk.edu

Received 14 January 2022; Revised 5 February 2022; Accepted 15 February 2022; Published 25 February 2022

Academic Editor: Palanivel Velmurugan

Copyright (c) 2022 Abdulsattar Abdullah Hamad et al. This is an open access article distributed under the Creative Commons Attribution License, which permits unrestricted use, distribution, and reproduction in any medium, provided the original work is properly cited.

\begin{abstract}
Every recognized hospital's patient management unit (PMU) has focused its efforts on improving clinical patient care, with a process approach, analyzing it from adult emergency overcrowding to prolonged stays in clinical services. It is generating many patients waiting for beds in the emergency service. The PMU does not have a business intelligence (BI) platform that provides information in real-time, generating a blind browsing problem. The purpose is to demonstrate the need for a BI platform using Artificial Intelligence (AI) to analyze in real-time the relevant information for decision making. The methodology consists of analyzing qualitative and quantitatively the statistics of the last three years, both from the emergency service and from the clinical services. This study shows that the saturation of the emergency service responds to the number of patients waiting for beds, which interferes with outpatient care. The projections for 2020 underestimated the demand, and the efforts to open hospital beds and home hospitalization quotas allowed to shovel said excess demand. The average stay numbers continue to increase, as does the number of hospitalized patients for emergencies, generating a progressive growth in demand. It is necessary to have a BI system adapted with AI to perform real-time analysis of the GRD, to be able to act during hospitalization and not afterward.
\end{abstract}

\section{Introduction}

Innovation is not an element that characterizes the public health sector-being able to incorporate cutting-edge technological tools in the BI area, such as AI, which allows data processing in real-time and not ex-post of the big data provided by the group related diagnostics (GRD) [1], will generate a radical change in the way of doing management clinic, much more proactive and focused on where the problem really lies. Having BI information in real-time, viewed by clinical heads and managers, will allow for more success when making decisions. The generation of quality data could reveal the need to incorporate professionals from the area of industrial engineering, logistics, computer science, among others, into the clinical management team, giving the possibility of forming a clinical operations center, a development unit of institutional intelligence.

Throughout history, hospital emergency services have become the gateway to hospitals. The initial objective of these units was the care of patients suffering from an acute or chronic decompensated pathology with a potential risk of life compromise. But they have evolved to the increase of banal consultations that can be resolved at the primary health care level 1 [2]. The sustained growth of care 
experienced has been called overcrowding (saturation or collapse). This oversaturation is given by the clear imbalance between the exaggerated demand and the limited supply delivered by the unit in question. This overcrowding and inadequate hospital emergency services are common in all developed countries. Multiple factors impact the increase in demand, the most relevant being the change in demographic and cultural characteristics and mainly the population's health status. Every day sicker, older, and younger patients consult. Independence, therefore, requires a greater number of benefits and care. The emergency services of 50 years ago had physical and functional limitations, without their own personnel, without leadership or direct supervision, and without clear objectives or performance and quality indicators [3]. They have constantly evolved, going from having a coordinator to a unit with a single head, the development of guides and protocols that help to direct its operation, the incorporation of expedited care pathways, risk categorization systems, structural improvements, among others, however, overcrowding continues, with the exception of the Scandinavian countries, probably due to having a solid primary care model and greater social awareness 4 . Since the formation of the PMU, measures and continuous improvement plans have been implemented, impacting to a greater or lesser extent on the management indicators of our hospital. Together with the DRM Unit (group related diagnoses) and the Management Control Unit, they are systematically monitored. In order to contextualize the problem, it is necessary to know some concepts: the GRD. This system dates back to the 70s, designed by Fetter and Thompson at Yale University, United States [4]. Demographic and clinical data make up the minimum basic data set, which must be extracted retrospectively from the clinical history of each patient, providing the information substrate for the GRD classification system, thus generating groups of similar patients, both in clinical and expense terms. By processing GRD data, a series of indicators were obtained that allow: epidemiological monitoring, clinical management, standardized comparison of hospital activity with other effectors or groups of these, hospital budgeting and planning, and the development and implementation of prospective payment systems. After visiting some health institutions in our country's capital city, Baghdad, which presented their business intelligence (BI) platforms, based on the big data provided by ICTs, the immense advantage is obtained when taking decisions and resource allocation in a hospital with respect to blind surfers. Data is generated exponentially on a daily basis, data that can often be irrelevant, but there are others that, with correct analysis, can generate a great impact in terms of management [5].

The GRD and this system's data are an important substrate for BI platforms. The HIS, LIS, and RIS-PACS are other important sources of information for these platforms. However, a great difficulty when analyzing HIS data is the large amount of information incorporated as free text, a series of unstructured data that makes it impossible to analyze it without the incorporation of more advanced computer tools, intelligence software artificial (AI), which manage to structure this information and analyze it together with the other structured data, ceasing to be big data and going on to be called smart data [6].

The PMU, since its formation, has tried to analyze clinical processes by collecting data and processing these, with an analysis focused on the processes and their systematization. However, this work has been carried out in a precarious and inefficient way, with data that may be misleading and/or irrelevant. Decision-making in relation to clinical management in this health facility is often based on unreliable data obtained from multiple sources, which are sometimes disparate. There are no technological tools for business intelligence, which allow cross-sectional information between the DRM, management control, patient management units, and the management team. Currently, there are clinical services that have average stay well above the standard of the norm, which generates efficiency in the use of resources, mainly, the bed resource, with the corresponding impact on the entire chain of production of services that provides a hospital to its beneficiaries. Let us look at the results of the entire hospital. They are very good, given that there are very efficient clinical units, thus improving the indicators of the other clinical services with worse efficiency.

As previously mentioned, the pediatric and gynecologicalobstetric services are not part of this analysis since their operation is optimal. However, the production line of health services that begins in the adult emergency service to medicine, surgery, ICU, and short psychiatric stay is the axis of greatest concern for the patient management unit, a process where there is a greater concentration of inefficiencies, both intrahospital and integrated health services networks. The reports delivered by the DRM and management control unit are published 15 days after the end of the month, at which time nothing can be done to improve the service and achieve better efficiency results. Currently, the head of the PMU and the respective heads of service (medicine and surgery) make joint visits, emphasizing those patients who have a stay equal to or greater than ten days, believing that they are those who prolong the average stay of the however, this biassed view of the process prevents us from being able to visualize whether the stays of patients with less than ten days conform to the standard of the norm, and these may be far outside the standard and it is necessary to have a BI system adapted with AI to perform real-time analysis of the GRD, to be able to act during hospitalization and not afterward. The general objective of this work is to demonstrate the need to incorporate advanced technological tools for business intelligence (AI, machine learning, smart data, and big data) [6] based on the GRD to the patient management unit to improve clinical management and decision-making based on data that adds value [6-8].

\section{Methodology}

A descriptive, qualitative, and quantitative analysis of the adult emergency service is carried out, for which the data is extracted from the hospital's medical history computer system, Trakcare in its 2013 version. The report called "GO urgent care book" must be downloaded with a periodicity not exceeding one month, due to the large amount of data that must be exported [7]. All these reports are exported 
in .csv files, which were opened with the Microsoft Office Professional Plus 2018 Excel software and compiled into an Excel file, with a .xlsx extension, with the name of "urgency database." This file contains information from the first day of February 2020 to the last day of December 2020. The database contains great details of the information of the consultations made in the adult emergency service of the government hospital. To protect the identity of patients and under the law on the rights and duties of patients, the database has been anonymized, erasing all data that relate them to identity. This database was tabulated, leaving only relevant information for this work, leaving the fields of "admission date," "admission time," "triage date," "triage time," "care date," "time attention," "discharge date," "discharge time," "waiting time for triage," "waiting time for attention," and "discharge waiting time." With this tabulated information, dynamic graphics are generated for further analysis. The number of patients hospitalized in the emergency service, in a condition of "awaiting a bed," was extracted from the Centralized Bed Management Unit platform, dependent on the Ministry of Health. The information downloaded from this site is processed in Excel, and graphics are generated for further analysis. Subsequently, we proceed with the analysis of the data provided by the GRD Unit of government general hospital; files of the type .xls are shared through free access folders on the Hospital computers. Two files are extracted, one with information for 2020 and 2019 and the other with 2019 and 2020. The information is compiled and tabulated using Excel software. Dynamic graphs are generated for subsequent interpretation of the data. These GRD data are divided into general hospital data and clinical service. For practical purposes, this work only refers to clinical services for adults, excluding gynaecology-obstetrics, medicine, surgery, short psychiatric stay, ICU, and emergency, applying the concepts available in the Literature.

\section{Results}

Since February 2018, when the adult emergency service began to use the HIS of the Intersystem Company, Trak care, crucial information has been collected when making decisions. The following Figure 1 shows the monthly behavior of inquiries from February 2018 to December 2020, with a linear downward trend. The seasonality of the behavior of the consultations can also be appreciated, with a paradoxical decrease in the winter months (Figure 1).

Suppose we project the data to month 1 of 2018, considering an average of consultations in the same month that information is available. In that case, we can see a decrease in the number of adult emergency consultations with a variation of $-5.54 \%$ in 2020 compared to the previous year, which is equivalent to 9 fewer patients per day.

Figure 2 shows the monthly behavior of the percentage of patient care in the same period as Figure 1. It is shown that knowing which the points of improvement and the measures were taken to increase the opportunity of care resulted in progressive effect improving the annual averages of care of $68.84 \%$ in $2018,75.37 \%$ in 2019 , and $81.50 \%$ in 2020. The measures taken were mainly to raise awareness among staff in improving the amount of care, in addition of some changes in the human resource staff (Figure 2).

The incorporation of extra nursing human resources, its sole and exclusive function being the categorization of patients, allowed the percentage of patients without categorization to be reduced to annual figures of $2.41 \%$, considering the initial figures around $5.1 \%$. This modification also greatly impacted the waiting times from admission to categorization, with a progressive decrease and maintenance of very good times (Figure 3 ).

The reinforcement of human medical resources, incorporated in the peak hours, revealed by the analysis of histograms of consultations (Figure 4) and allowed to a great extent to increase the opportunity of attention. In addition, to reduce the waiting times for attention from the triage, with averages in 2018 of 2.63 hours, reaching an average of 2.09 hours in 2020 , with a percentage decrease at the end of 2019 of $13 \%$ compared to 2018 and 2020 with a decrease of $8 \%$ compared to its predecessor. The duration of care also showed a decrease from the averages, but only in 2019, with a decrease from 2.72 to 2.43 hours (variation $11 \%$ ); however, the figures for 2020 show an average of 2.47 , with a slight increase of $2 \%$.

As evidenced in the previous figures, all the efforts made to improve processes in the adult emergency service have generated a positive impact on the statistics. However, this is not enough, given that the number of patients hospitalized in the emergency room, waiting for a bed in clinical services, has increased linearly, as can be seen in the following Figure 5.

It is worth mentioning that during the year 2020, 2 important events have occurred in the Teaching Hospital Baghdad, Iraq, the first, a significant increase in the number of patients in the Home Hospitalization Unit, from 15 patients to 65 (growth of 33.3\%). The other relevant fact, as far as clinical management is concerned, was the opening of the Middle Care Unit, with a gain of 9 beds ( 24 beds were opened, but during construction, 15 extra places were worked in home hospitalization), and 3 beds from the Obstetric Gynaecology Service that were transferred to the surgery service, adding a total of 62 hospitalization places. If the start-up time of these hospitalization units is weighted, we can see an increase of 7.230 bed days, with an average stay of 12.78 days, with an approximate of almost 3 patients per day. It is worth saying that, without this intervention in the increase in hospitalization quotas, the emergency room would maintain an average of patients waiting for bed over 40. The following Figure 6 shows the gradual increase in hospitalization quotas.

With this increase in quotas, it would be expected that the number of patients waiting for beds in the emergency service would decrease, however, the opposite occurs, as we saw previously, it increased to 37 patients per day on average. To avoid bias in the analysis of this information, when working only with the annual average, Figure 6 shows the behavior of the weekly averages of patients waiting for beds in the adult emergency service.

We see a drop in values in the last weeks, coinciding with the opening of quotas in the last 14 weeks, with an average of 


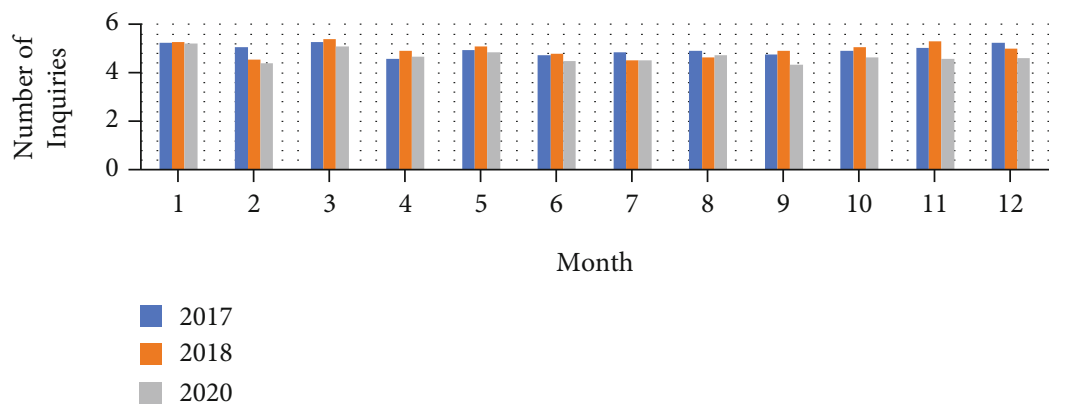

FigURE 1: Number of monthly consultations grouped by year of the adult emergency service.

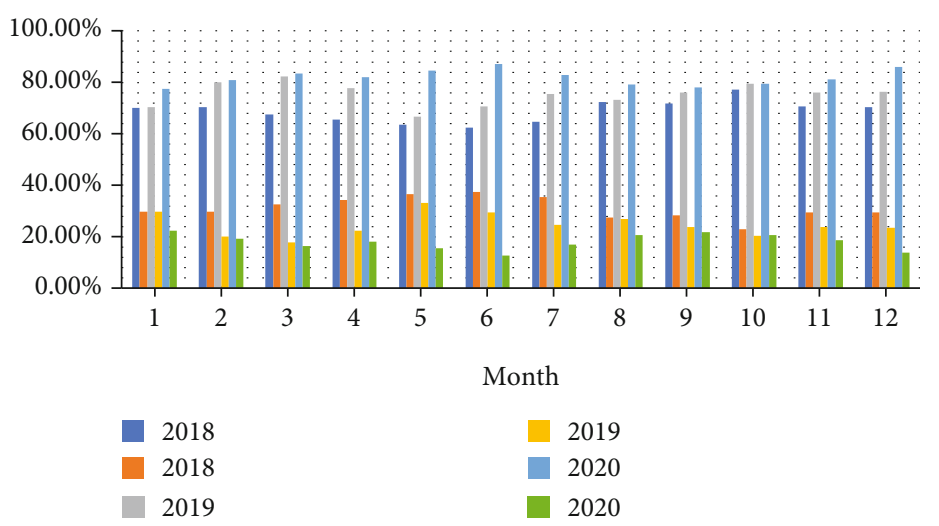

FigURE 2: Percentage of patients treated vs. not treated, grouped by month and year.

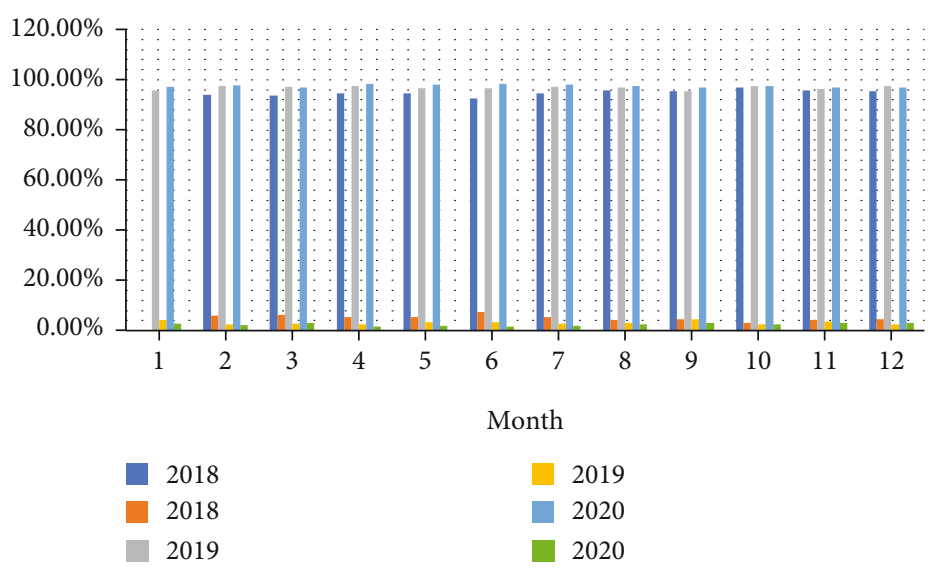

Figure 3: Percentages of patients with and without triage grouped by month and year.

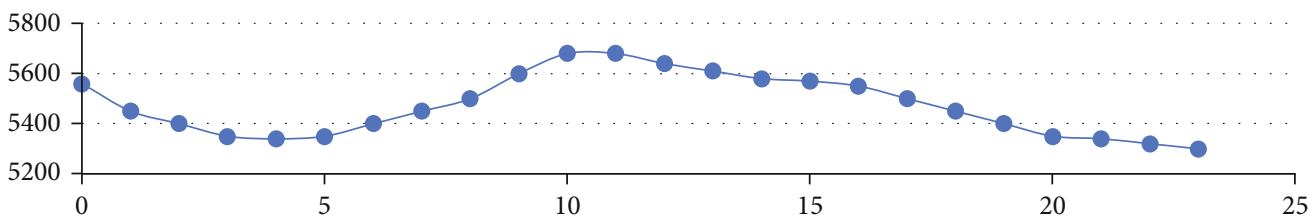

FIGURE 4: Histogram of hourly distribution of queries. 


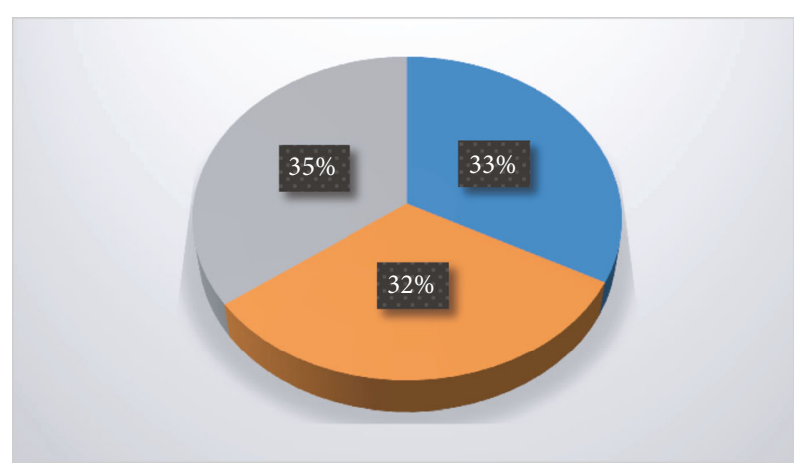

FIGURE 5: Daily average patients waiting for beds.

35 patients/day. The average number of patients from the first part of the year to week 36 had an average of 38 patients/day, with a peak of 47 at week 36 . The dotted red line corresponds to a moving average trend line that considers 12 periods ( 12 weeks). Let us analyze the number of patients hospitalized by the emergency service, the main entrance door to the hospital. We can see a small variation if we compare the number of admissions, and the trend line of the following Figure 7 is almost flat.

If we project the data to January 2018 and make an annual comparison, we can see that the variation between 2019 and 2020 is 0.77 more patients per day, similar to that of 2018. So, we have an increase in the capacity to hospitalize patients. The number of hospitalizations has a minimal increase compared to the previous year and almost the same as in 2018. Still, the number of patients waiting for beds in the emergency service has increased linearly concerning their predecessors. In the following Figure 8, we will review the behavior of the refined average stay, and this data excludes pediatric services, gynaecology-obstetrics, and the pension service, given that their source of income for patients comes from another route, which is not the service adult emergency. In the figure above, we can see a clear upward trend in average stays, with variations of $1.55 \%$ and $1.28 \%$, respectively. The following figure shows in more detail the mean stays per service with the greatest impact in terms of adult patients. Therefore, this increase in MS, considering that the amount of admissions through the adult emergency service has increased minimally (1.2\% if we compare 2020 with 2018 and $4.87 \%$ if we do it with 2019) and hospitalization quotas increased by $39 \%$. Does this explain why the number of patients waiting for a bed in the adult emergency service has increased? If we now see the number of expenditures in the same period comparing the last 3 years, a significant increase stands out in 2020, around 5\%, compared to the previous ones, as we can see in the following Figure 8.

The following Figure 9 shows that the variation between 2018 and 2019 is insignificant, however, for 2020, if a significant increase in the number of discharges is observed, which, added to the increase in the average stay, gives us as a product an increase in the number of stays close to 8,600 .

As mentioned above, this increase in hospitalization quotas, whether beds or home hospitalization quotas, gener- ates capacity for some 7,230 more stays for 2020. With 1,370 stays per year without hospitalization quota, which, added to the increase in the number of hospitalizations by the emergency service, about 280 more patients per year, resulting in about 3,500 more stays. This number, divided by 365 days of the year and by the MS cleared of 12.58, is equivalent to approximately 1 more patient/day, which would not explain the increase in patients waiting for beds in the emergency department, going from an average from 34 to 37 patients/ day in 2020. Surgical activity in 2020 had an increase compared to 2019, reaching 650 more patients for the cut-off of October 2020, which is equivalent to 1.78 more patients (2.13 more patients if we project the information linearly), which explains the other 2 patients missing to complete the 37 patients/day on average that the urgency keeps awaiting beds. We can see that the medical service maintains a $40 \%$ inefficiency concerning the norm, surgery has managed to drop to only $10 \%$ inefficiency, the short-stay psychiatric unit exceeds twice the length of stay corresponding to the norm, and the UTI failed to improve $36 \%$. The ICU is the only one with below 1.0 in the last two years see the Table 1 .

Some of the identified biases are mentioned below:

(a) When analyzing the data, it is the route of entry from the OB-GYN service to UPC and vice versa, which are not registered in these calculations, we work under the assumption that their impact is marginal

(b) Given that this analysis is carried out on the annual average of refined stays, there is also an unseen variation when viewing the results

(c) The seasonality of the demand is not considered in this study either

(d) The variation in the number of beds and home hospitalization quotas, whose incorporation was gradual during the last months of 2020, was partially considered in some of the estimates

\section{Discussion}

A series of successful decisions were implemented in the emergency service of Government hospital, Baghdad, supported and based on solid statistics collected over the last 3 years. These actions were implemented little by little, and that is how they also left seeing satisfactory results. However, despite all efforts to continue improving, no better results have been achieved. This improvement curve has already become asymptotic, where for more resources that are introduced, the results will not improve proportionally. The problem lies in the rest of the hospital, in the clinical services that maintain high average stay levels, understanding the causes of this problem is crucial when making decisions.

The patient management unit has made great efforts to understand the data and make strategic moves of great impact, without achieving it satisfactorily. The results of this work have allowed a more detailed look at the clinical management process, based on the data provided by the GRD, undoubtedly an advance of what can be achieved in much 

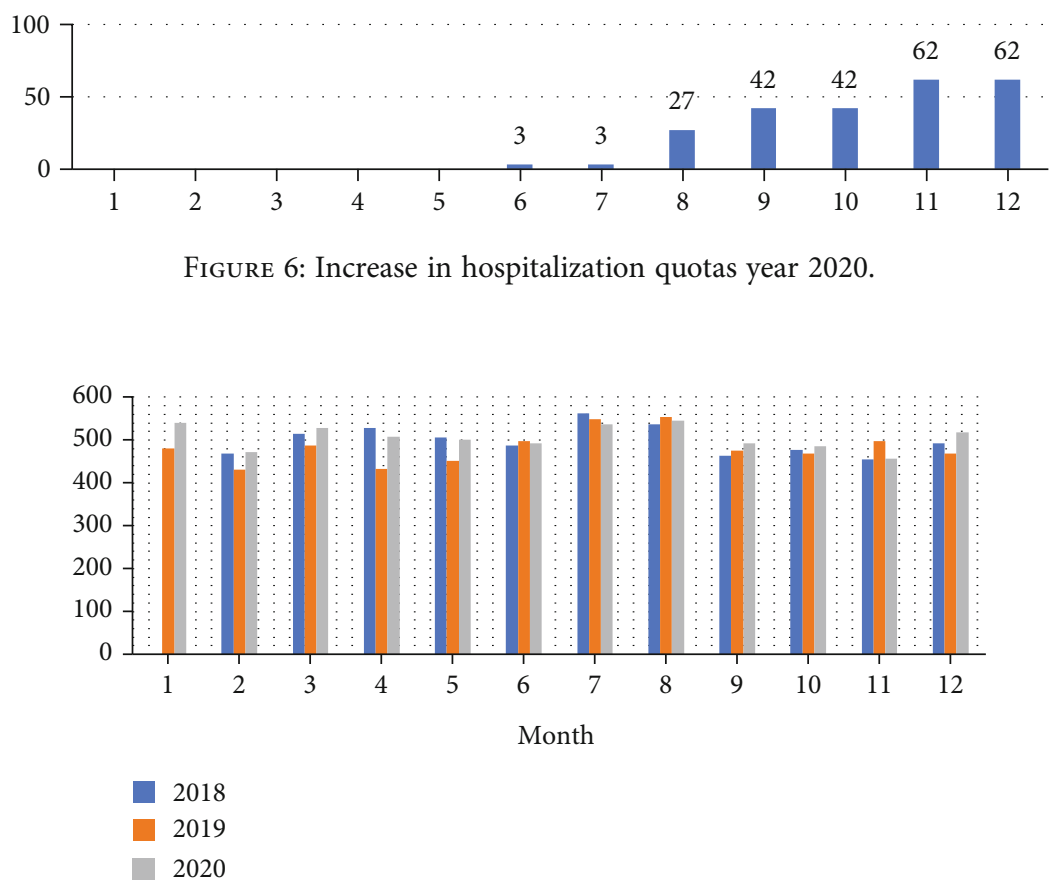

FIGURE 7: Number of monthly hospitalizations grouped by year in the emergency service.

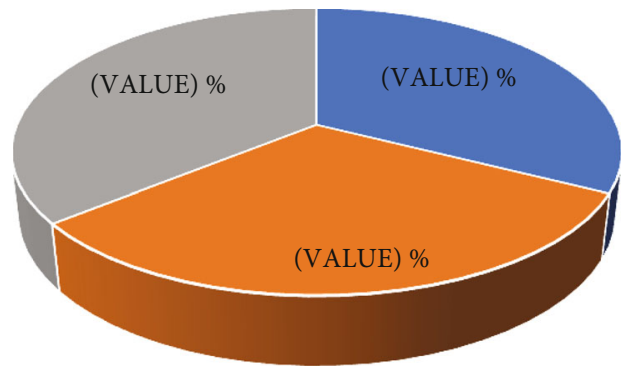

Figure 8: Total expenditures per year.

more detail when using technological development tools with artificial intelligence, searching for data that adds value to decision-making, data that smart data can deliver.

The results of this work show that the efforts have not generated any impact on the reduction of the average stay; on the contrary, this indicator has been increasing. Still, when looking at the global data of the hospital, we see that the indicators are good, generating an equivocal degree of satisfaction. At the end of 2019, the PMU predicted that the number of patients waiting for hospital beds in the emergency service for 2020 would be 38 patients per day on average, based on a linear projection of data from previous years. The results were slightly lower, averaging 37 patients per day. But, it must be remembered that the number of beds and places in the home hospitalization unit had a relevant growth, increasing by 62 places in total. This increase in quotas was put into operation in the last months of 2020, generating a buffer for the increase in demand, reducing the average to 32 patients per day in the last 8 weeks and 31 in the last 4 weeks. This allows us to start 2019 on a better footing. However, efforts must focus on improving the average stay of the affected services. Even finer data can be obtained, however, this involves a lot of artisan work, using simple Excel tools, downloading data, compiling, and tabulating precariously [9].

The implementation of artificial intelligence and machine learning in the HIS of Government hospital, Baghdad, as a solution to the problem posed, which structures the data entered in an unstructured way, as free text in the clinical file, together with the structured data provided by the system, they would generate the partial MBDS for each hospitalization episode, thus allowing data on each hospitalized patient to be available every day and processed by the GRD algorithm. This processing will give us results for each patient with a GRD weight calculated on the data entered up to then, a weight that will allow us to project and compare, with the standard of the norm, the days of stay that should remain in the hospital. This will provide fine information to the patient management unit, who, together with the respective service manager, will closely review the cases in which their estimated average stay has been met, to expeditiously manage the missing requirements to decide their discharge, or add those diagnoses or procedures that could have been performed. Still, there is no record of it in the computer system.

This real-time analysis of the GRD would also allow us, with machine learning, to train the machine to generate sufficient and necessary algorithms for the coding of cases, which in the future will allow us to have the information with just a couple of days' time lag and without the need for specialized human resources, automating time-dependent processes. These officials should be reassigned to DRM analysis functions, with a clinical focus on efficiency. 


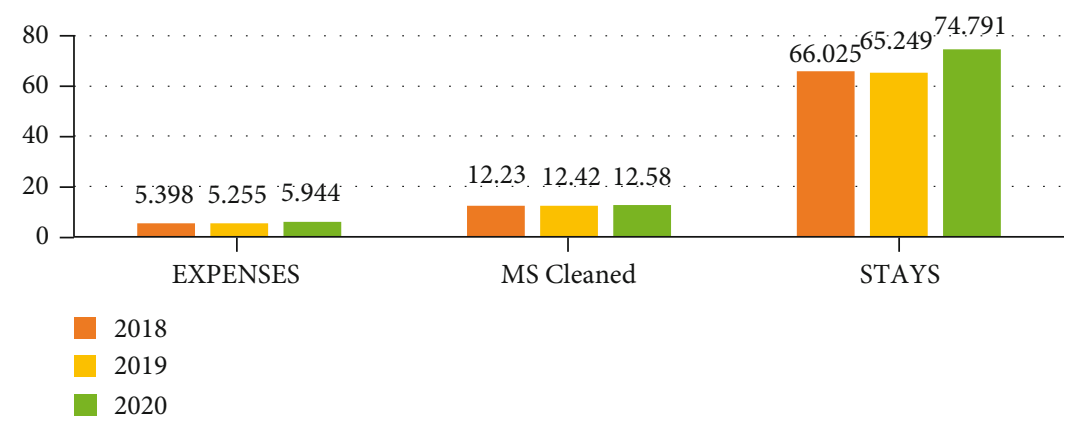

Figure 9: Variation of discharges between 2018 and 2020 in (\%).

TABLE 1: Average weight average GRD.

\begin{tabular}{lccccccc}
\hline Year & Medicine & Surgery & Short stay & ICU & UTI & Adult emergency unit & Grand total \\
\hline 2018 & 10707 & 10890 & 0.5777 & 43112 & 14812 & 0.7794 & 15515 \\
2019 & 11917 & 11442 & 0.5439 & 48526 & 20035 & 0.8085 \\
2020 & 11285 & 11892 & 0.5871 & 45375 & 20835 & 0.7528 & 17574 \\
Grand total & 11303 & 11408 & 0.5695 & 45671 & 18561 & 0.7802 & 17131 \\
\hline
\end{tabular}

\section{Conclusions}

The knowledge of historical data has allowed the emergency service to make the right decisions when deciding where to implement improvements and move the pieces strategically to achieve a greater impact on management. There are still things to be done, however, due to the large amount of data available, it is becoming increasingly difficult to process them in an artisanal way. Until now, the patient management unit navigates almost blindly, trying to hit the mark within the nebula, despite this, it has been able to improve very important processes within the day-to-day clinical management, however, the average stay is growing more and more and more, greatly impacting the contingency measures implemented, such as the increase in the number of beds and places in the home hospitalization unit.

Power is obtained by knowing the data, to know the data, tools are needed to help you understand them, which is why after this sequential analysis of the care process or this production line of clinical services, we can recommend that the incorporation of technological tools of the artificial intelligence type, to achieve a finished data processing, necessary for decision making. The GRD and the data provided by this unit must be treated in a better way, carrying out a multidisciplinary analysis of their behavior, to improve centrally the problems of clinical management, generating the need to incorporate professionals who normally do not perform their functions in the health sector due to lack of positions, with this, it is necessary to form a Unit or Committee for Institutional Development and Intelligence [10-12].

Integration with the financial team is necessary to perform efficiency analysis about clinical processes, which are often trapped by complementary examinations, which often have lower costs than what is spent on the waiting stay or complications that it can present, which, at times, can cost the life of the patient. Technology is here to stay; it is neces- sary to keep up with technological development to not be left behind. It is necessary to renew health in our country, which has maintained the same business model for many years, there are only technological advances in examinations and treatments, but the functional structure is still very conservative, and it is necessary to incorporate disruptive ideas at the time of taking of decisions.

\section{Data Availability}

The data underlying the results presented in the study are available within the manuscript.

\section{Conflicts of Interest}

The authors declare that they have no conflicts of interest regarding the publication of this paper.

\section{References}

[1] P. K. Pramanik, S. Pal, and M. Mukhopadhyay, "Healthcare big data: A comprehensive overview," in Research Anthology on Big Data Analytics, Architectures, and Applications, IGI Global, 2018.

[2] S. Glasser, "Research Methodology for Studies of Diagnostic Tests," in Essentials of Clinical Research, pp. 313-326, Springer, Cham, 2008.

[3] J. Liu, W. Wang, F. Ma, Y. B. Yang, and C. S. Yang, "A datamodel-fusion prognostic framework for dynamic system state forecasting," Engineering Applications of Artificial Intelligence, vol. 25, no. 4, pp. 814-823, 2012.

[4] M. Lungen, B. Dredge, A. Rose, R. Corey, E. Plamper, and K. Lauterbach, "Using diagnosis-related groups. The situation in the United Kingdom National Health Service and in Germany," The European journal of health economics: HEPAC: health economics in prevention and care, vol. 5, pp. 287-289, 2005. 
[5] L. Hong, M. Luo, R. Wang, P. Lu, W. Lu, and L. Lu, "Big data in health care: applications and challenges," Data and Information Management, vol. 2, no. 3, pp. 175-197, 2019.

[6] C. A. Laurentys, R. M. Palhares, and W. M. Caminhas, "Design of an artificial immune system based on danger model for fault detection," Expert Systems with Applications, vol. 37, no. 7, pp. 5145-5152, 2010.

[7] P. O. Ouma, J. Maina, P. N. Thuranira et al., "Access to emergency hospital care provided by the public sector in subSaharan Africa in 2015: a geocoded inventory and spatial analysis," The Lancet Global Health, vol. 6, no. 3, pp. e342-e350, 2018.

[8] A. A. Hamad, M. L. Thivagar, J. Alshudukhi et al., "Secure complex systems: a dynamic model in the synchronization," Computational Intelligence and Neuroscience, vol. 2021, Article ID 9719413, 6 pages, 2021.

[9] S. Rouhani, A. Ashrafi, A. Zareravasan, and S. Afshari, "The impact model of business intelligence on decision support and organizational benefits," Journal of Enterprise Information Management, vol. 29, no. 1, pp. 19-50, 2016.

[10] A. A. Hamad, M. L. Thivagar, and K. M. Sagayam, "Analysis of some topological nodes using the adaptive control based on 9D, hypothesis theoretical to COVID-19," International Journal of Computer Applications in Technology, vol. 66, no. 3/4, pp. 241-245, 2021.

[11] V. Sriram, G. Gururaj, and A. Hyder, "Public-private implementation of integrated emergency response services: case study of GVK Emergency Management and Research Institute in Karnataka, India," Surgery, vol. 162, no. 6S, pp. S63-S76, 2017.

[12] I. Harvey, "Artificial evolution and real robots," Artificial Life and Robotics, vol. 1, no. 1, pp. 35-38, 1997. 